\title{
MIMO Radar Ambiguity Properties and Optimization Using Frequency-Hopping Waveforms
}

\author{
Chun-Yang Chen, Student Member, IEEE, and P. P. Vaidyanathan, Fellow, IEEE
}

\begin{abstract}
The concept of multiple-input multiple-output (MIMO) radars has drawn considerable attention recently. Unlike the traditional single-input multiple-output (SIMO) radar which emits coherent waveforms to form a focused beam, the MIMO radar can transmit orthogonal (or incoherent) waveforms. These waveforms can be used to increase the system spatial resolution. The waveforms also affect the range and Doppler resolution. In traditional (SIMO) radars, the ambiguity function of the transmitted pulse characterizes the compromise between range and Doppler resolutions. It is a major tool for studying and analyzing radar signals. Recently, the idea of ambiguity function has been extended to the case of MIMO radar. In this paper, some mathematical properties of the MIMO radar ambiguity function are first derived. These properties provide some insights into the MIMO radar waveform design. Then a new algorithm for designing the orthogonal frequency-hopping waveforms is proposed. This algorithm reduces the sidelobes in the corresponding MIMO radar ambiguity function and makes the energy of the ambiguity function spread evenly in the range and angular dimensions.
\end{abstract}

Index Terms-Ambiguity function, frequency-hopping codes, linear frequency modulation (LFM), MIMO radar, simulated annealing, waveform design.

\section{INTRODUCTION}

I $\mathrm{N}$ the traditional single-input multiple-output (SIMO) radar, the system can only transmit scaled versions of a single waveform. The multiple-input multiple-output (MIMO) radar system allows transmitting orthogonal (or incoherent) waveforms in each of the transmitting antennas [1], [2]. These waveforms can be extracted by a set of matched filters in the receiver. Each of the extracted components contains the information of an individual transmitting path. There are two different kinds of approaches for using this information. First, the spatial diversity can be increased. In this scenario, the transmitting antenna elements are widely separated such that each views a different aspect of the target. Consequently, the target radar cross sections (RCS) are independent random variables for different transmitting paths. Therefore, each of the components extracted by the matched filters in the receiver contains independent information about the target. Since we can obtain multiple independent measurements about the target, a better detection performance can be obtained

Manuscript received February 04, 2008; revised July 20, 2008. First published August 19, 2008; current version published November 19, 2008. The associate editor coordinating the review of this manuscript and approving it for publication was Dr. Chong-Meng Samson See. This work was supported in parts by the ONR Grant N00014-06-1-0011, and the California Institute of Technology, Pasadena.

The authors are with California Institute of Technology, Pasadena, CA 91125 USA (e-mail: cyc@caltech.edu; ppvnath@ systems.caltech.edu).

Digital Object Identifier 10.1109/TSP.2008.929658
[3]-[5]. Second, a better spatial resolution can be obtained. In this scenario, the transmitting antennas are colocated such that the RCS observed by each transmitting path are identical. The components extracted by the matched filters in each receiving antennas contain the information of a transmitting path from one of the transmitting antenna elements to one of the receiving antenna elements. By using the information about all of the transmitting paths, a better spatial resolution can be obtained. It has been shown that this kind of radar system has many advantages such as excellent interference rejection capability [9], [10], improved parameter identifiability [8], and enhanced flexibility for transmit beampattern design [11], [12]. Some of the recent work on the colocated MIMO radar has been reviewed in [7]. In this paper, we focus on the colocated MIMO radar.

Recently, several papers have been published on the topic of MIMO radar waveform design [11]-[15]. In [11], the covariance matrix of the transmitted waveforms has been designed to form a focused beam such that the power can be transmitted to a desired range of angles. In [12], the authors have also focused on the design of the covariance matrix to control the spatial power. However, in [12], the cross correlation between the transmitted signals at a number of given target locations is minimized. In [13]-[16], unlike [11] and [12], the entire waveforms have been considered instead of just the covariance matrix. Consequently these design methods involve not only the spatial domain but also the range domain. These methods assume some prior knowledge of the impulse response of the target and use this knowledge to choose the waveforms which optimize the mutual information between the received signals and the impulse response of the target. The waveform design which uses prior knowledge about the target has been done in the traditional SIMO radar system as well [17]. In this paper, we consider a different aspect of the waveform design problem. We design the waveforms to optimize the MIMO radar ambiguity function [6]. Unlike the above methods, we do not assume the prior knowledge about the target.

The waveform design problem based on optimization of the ambiguity function in the traditional SIMO radar has been well studied. Several waveform design methods have been proposed to meet different resolution requirements. These methods can be found in [25] and the references therein. In the traditional SIMO radar system, the radar receiver uses a matched filter to extract the target signal from thermal noise. Consequently, the resolution of the radar system is determined by the response to a point target in the matched filter output. Such a response can be characterized by a function called the ambiguity function [25]. Recently, San Antonio et al. [6] have extended the radar ambiguity function to the MIMO radar case. It turns out that the radar waveforms affect not only the range and Doppler resolution but 
also the angular resolution. It is well-known that the radar ambiguity function satisfies some properties such as constant energy and symmetry with respect to the origin [25]. These properties are very handy tools for designing and analyzing the radar waveforms. In this paper, we derive the corresponding properties for the MIMO radar case.

The major contributions in this paper are twofold: 1) to derive new mathematical properties of the MIMO ambiguity function, and 2) to design a set of frequency-hopping pulses to optimize the MIMO ambiguity function. The MIMO radar ambiguity function characterizes the resolutions of the radar system. By choosing different waveforms, we obtain a different MIMO ambiguity function. Therefore, the MIMO radar waveform design problem is to choose a set of waveforms which provides a desirable MIMO ambiguity function. Directly optimizing the waveforms requires techniques such as calculus of variation. In general this can be very hard to solve. Instead of directly designing the waveforms, we can impose some structures on the waveforms and design the parameters of the waveforms.

As an example of this idea, the pulse waveforms generated by frequency-hopping codes are considered in this paper. Frequency-hopping signals are good candidates for the radar waveforms because they are easily generated and have constant modulus. In the traditional SIMO radar, Costas codes [20], [21] have been introduced to reduce the sidelobe in the radar ambiguity function. The frequency-hopping waveforms proposed in [19] have been applied in a MIMO High-Frequency Over The Horizon (HF OTH) radar system [18]. The frequency-hopping waveforms proposed in [19] are originally designed for multiuser radar system. The peaks in the cross correlation functions of the waveforms are approximately minimized by the codes designed in [19]. However, in the multiuser scenario, each user operates its own radar system. This is different from the MIMO radar system where the receiving antennas can cooperate to resolve the target parameters. In this paper, we design the frequency-hopping waveforms to optimize the MIMO ambiguity function which directly relates to the MIMO radar system resolution.

The rest of the paper is organized as follows. In Section II, the MIMO radar ambiguity function will be briefly reviewed. Section III derives the properties of the MIMO radar ambiguity function. In Section IV, we derive the MIMO radar ambiguity function when the pulse trains are transmitted. In Section V, we define the frequency-hopping pulse waveforms in MIMO radar and derive the corresponding MIMO ambiguity function. In Section VI, we formulate the frequency-hopping code optimization problem and show how to solve it. In Section VII, we test the proposed method and compare its ambiguity function with the LFM (linear frequency modulation) waveforms. Finally, Section VIII concludes the paper. The results in this paper are for uniform linear arrays but they can easily be generalized.

\section{REVIEW OF MIMO RADAR AMBIGUITY FUNCTION}

In a SIMO radar system, the radar ambiguity function is defined as [25]

$$
|\chi(\tau, \nu)| \triangleq\left|\int_{-\infty}^{\infty} u(t) u^{*}(t+\tau) e^{j 2 \pi \nu t} d t\right|
$$

(a)

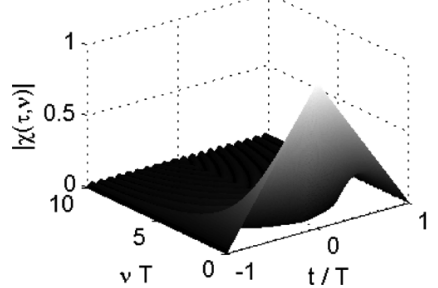

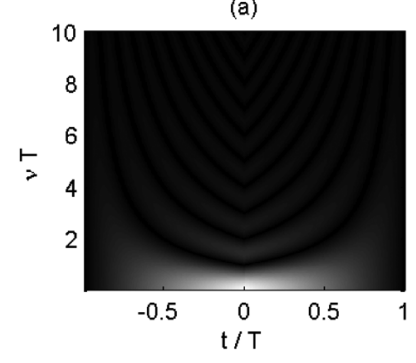

(b)
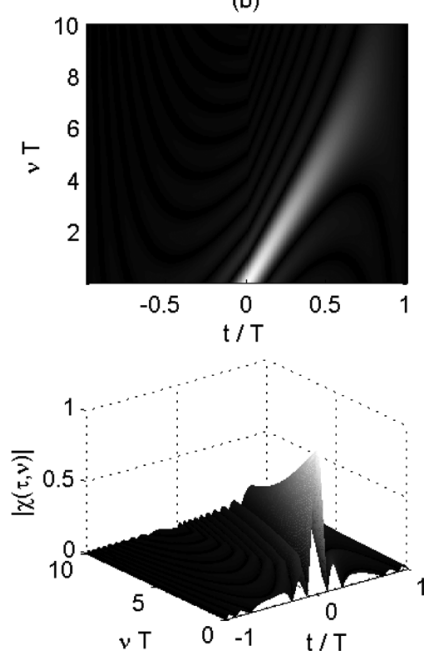

Fig. 1. Examples of ambiguity functions: (a) Rectangular pulse, and (b) Linear frequency modulation (LFM) pulse with time-bandwidth product 10, where $T$ is the pulse duration.

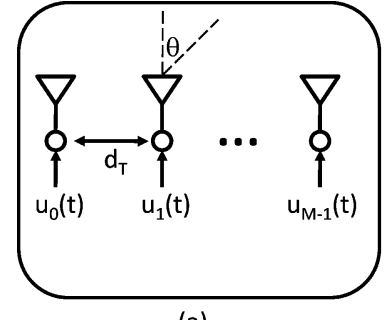

(a)

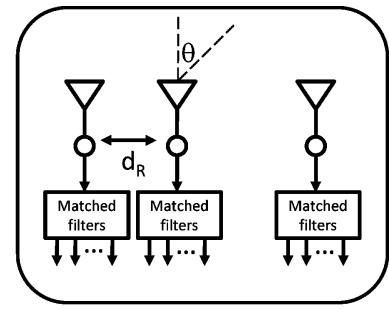

(b)
Fig. 2. MIMO radar scheme: (a) transmitter and (b) receiver.

where $u(t)$ is the radar waveform. This two-dimensional function indicates the matched filter output in the receiver when a delay mismatch $\tau$ and a Doppler mismatch $\nu$ occur. The value $|\chi(0,0)|$ represents the matched filter output without any mismatch. Therefore, the sharper the function $|\chi(\tau, \nu)|$ around $(0$, $0)$, the better the Doppler and range resolution. Fig. 1 shows two examples of the ambiguity function. These two ambiguity functions show different Doppler and range tradeoffs. One can see that the LFM pulse has a better range resolution along the cut where Doppler frequency is zero.

The idea of radar ambiguity functions has been extended to the MIMO radar by San Antonio et al. [6]. In this section, we will briefly review the definition of MIMO radar ambiguity functions. We will focus only on the uniform linear array (ULA) case as shown in Fig. 2. The derivation of the MIMO ambiguity function for arbitrary array can be found in [6]. We assume the transmitter and the receiver are parallel and colocated ULAs. The spacing between the transmitting elements is $d_{T}$ and the spacing between the receiving elements is $d_{R}$. The function $u_{i}(t)$ indicates the radar waveform emitted by the $i$ th transmitter.

Consider a target at $(\tau, \nu, f)$ where $\tau$ is the delay corresponding to the target range, $\nu$ is the Doppler frequency of the target, and $f$ is the normalized spatial frequency of the target defined as

$$
f \triangleq 2 \pi \frac{d_{R}}{\lambda} \sin \theta
$$


where $\theta$ is the angle of the target and $\lambda$ is the wavelength. The demodulated target response in the $n$th antenna is proportional to

$$
y_{n}^{\tau, \nu, f}(t) \approx \sum_{m=0}^{M-1} u_{m}(t-\tau) e^{j 2 \pi \nu t} e^{j 2 \pi f(\gamma m+n)}
$$

for $n=0,1, \ldots, N-1$, where $N$ is the number of receiving antennas, $u_{m}(t)$ is the radar waveform emitted by the $m$ th antenna, $\gamma \triangleq d_{T} / d_{R}$, and $M$ is the number of transmitting antennas. If the receiver tries to capture this target signal with a matched filter with the assumed parameters $\left(\tau^{\prime}, \nu^{\prime}, f^{\prime}\right)$ then the matched filter output becomes the equation shown at the bottom of the page. The first part in the right hand side of the equation represents the spatial processing in the receiver, and it is not affected by the waveforms $\left\{u_{m}(t)\right\}$. The second part in the right-hand side of the equation indicates how the waveforms $\left\{u_{m}(t)\right\}$ affect the spatial, Doppler, and range resolutions of the radar system. Therefore, we define the MIMO radar ambiguity function as

$$
\chi\left(\tau, \nu, f, f^{\prime}\right) \triangleq \sum_{m=0}^{M-1} \sum_{m^{\prime}=0}^{M-1} \chi_{m, m^{\prime}}(\tau, \nu) e^{j 2 \pi\left(f m-f^{\prime} m^{\prime}\right) \gamma}
$$

where

$$
\chi_{m, m^{\prime}}(\tau, \nu) \triangleq \int_{-\infty}^{\infty} u_{m}(t) u_{m^{\prime}}^{*}(t+\tau) e^{j 2 \pi \nu t} d t .
$$

Note that the MIMO radar ambiguity function can not be expressed as a function of the difference of the spatial frequencies, namely $f-f^{\prime}$. Therefore, we need both the target spatial frequency $f$ and the assumed spatial frequency $f^{\prime}$ to represent the spatial mismatch. We call the function $\chi_{m, m^{\prime}}(\tau, \nu)$ the cross ambiguity function because it is similar to the SIMO ambiguity function defined in (1) except it involves two waveforms $u_{m}(t)$ and $u_{m^{\prime}}(t)$. Fixing $\tau$ and $\nu$ in (2), one can view the ambiguity function as a scaled two-dimensional Fourier transform of the cross ambiguity function $\chi_{m, m^{\prime}}(\tau, \nu)$ on the parameters $m$ and $m^{\prime}$. The value $|\chi(0,0, f, f)|$ represents the matched filter output without mismatch. Therefore, the sharper the function $\left|\chi\left(\tau, \nu, f, f^{\prime}\right)\right|$ around the line $\{(0,0, f, f)\}$, the better the radar system resolution.

\section{PROPERTIES OF THE MIMO RADAR AMBIGUITY FUNCTION FOR ULA}

We now derive some new properties of the MIMO radar ambiguity function defined in (2). The properties are similar to some of the properties of the SIMO ambiguity functions (e.g., see [25]). We normalize the energy of the transmitted waveform to unity. That is,

$$
\int_{-\infty}^{\infty}\left|u_{m}(t)\right|^{2} d t=1 \quad \forall m .
$$

The following property characterizes the ambiguity function when there exists no mismatch.

1) Property 1: If $\int_{-\infty}^{\infty} u_{m}(t) u_{m^{\prime}}^{*}(t) d t=\delta_{m, m^{\prime}}$, then

$$
\chi(0,0, f, f)=M \quad \forall f .
$$

Proof: We have

$$
\chi_{m, m^{\prime}}(0,0)=\int_{-\infty}^{\infty} u_{m}(t) u_{m^{\prime}}^{*}(t) d t=\delta_{m, m^{\prime}} .
$$

Substituting the above equation into (2), we obtain

$$
\chi(0,0, f, f)=\sum_{m=0}^{M-1} \sum_{m^{\prime}=0}^{M-1} \delta_{m, m^{\prime}} e^{j 2 \pi \gamma\left(f m-f m^{\prime}\right)}=\sum_{m=0}^{M-1} e^{j 0}=M .
$$

This property says that if the waveforms are orthogonal, the ambiguity function is a constant along the line $\{(0,0, f, f)\}$ which is independent of the waveforms $\left\{u_{m}(t)\right\}$. This means the matched filter output is always a constant independent of the waveforms, when there exists no mismatch.

The following property characterizes the integration of the MIMO radar ambiguity function along the line $\{0,0, f, f\}$ even when the waveforms are not orthogonal.

2) Property 2:

$$
\chi(0,0, f, f) \geq 0
$$

and if $\gamma$ is an integer, then

$$
\int_{0}^{1} \chi(0,0, f, f) d f=M .
$$

Proof: By using the definitions in (2) and (3), we have

$$
\chi(0,0, f, f)=\int_{-\infty}^{\infty}\left|\sum_{m=0}^{M-1} u_{m}(t) e^{j 2 \pi f m \gamma}\right|^{2} d t \geq 0
$$

By using the definitions in (2) and (3) and changing variables, we obtain

$$
\begin{aligned}
\int_{0}^{1} \chi(0,0, f, f) d f & =\int_{0}^{1} \sum_{m=0}^{M-1} \sum_{m^{\prime}=0}^{M-1} \chi_{m, m^{\prime}}(0,0) e^{j 2 \pi f \gamma\left(m-m^{\prime}\right)} d f \\
& =\sum_{m=0}^{M-1} \sum_{m^{\prime}=0}^{M-1} \chi_{m, m^{\prime}}(0,0) \delta_{m, m^{\prime}}=M .
\end{aligned}
$$

$$
\begin{aligned}
& \sum_{n=0}^{N-1} \int_{-\infty}^{\infty} y_{n}^{\tau, \nu, f}(t) \cdot\left(y_{n}^{\tau^{\prime}, \nu^{\prime}, f^{\prime}}\right)^{*}(t) d t \\
& \quad=\left(\sum_{n=0}^{N-1} e^{j 2 \pi\left(f-f^{\prime}\right) n}\right) \cdot\left(\sum_{m=0}^{M-1} \sum_{m^{\prime}=0}^{M-1} \int_{-\infty}^{\infty} u_{m}(t-\tau) u_{m^{\prime}}^{*}\left(t-\tau^{\prime}\right) e^{j 2 \pi\left(\nu-\nu^{\prime}\right) t} d t \cdot e^{j 2 \pi\left(f m-f^{\prime} m^{\prime}\right) \gamma}\right) .
\end{aligned}
$$


This property says that when $\gamma$ is an integer, the integration of the MIMO radar ambiguity function along the line $\{0,0, f, f\}$ is a constant, no matter how waveforms are chosen. The following property characterizes the energy of the cross ambiguity function.

3) Property 3:

$$
\int_{-\infty}^{\infty} \int_{-\infty}^{\infty}\left|\chi_{m, m^{\prime}}(\tau, \nu)\right|^{2} d \tau d \nu=1
$$

Proof: We have

$$
\begin{aligned}
\int_{-\infty}^{\infty} & \int_{-\infty}^{\infty}\left|\chi_{m, m^{\prime}}(\tau, \nu)\right|^{2} d \tau d \nu \\
& =\int_{-\infty}^{\infty} \int_{-\infty}^{\infty}\left|\int_{-\infty}^{\infty} u_{m}(t) u_{m^{\prime}}^{*}(t+\tau) e^{j 2 \pi \nu t} d t\right|^{2} d \nu d \tau \\
& =\int_{-\infty}^{\infty} \int_{-\infty}^{\infty}\left|u_{m}(t) u_{m^{\prime}}^{*}(t+\tau)\right|^{2} d t d \tau
\end{aligned}
$$

where we have used Parseval's theorem [26] to obtain the last equality. By changing variables, we obtain

$$
\begin{aligned}
\int_{-\infty}^{\infty} \int_{-\infty}^{\infty} \mid u_{m}(t) u_{m^{\prime}}^{*} & \left.(t+\tau)\right|^{2} d t d \tau \\
& =\int_{-\infty}^{\infty}\left|u_{m}(t)\right|^{2} d t \int_{-\infty}^{\infty}\left|u_{m^{\prime}}(t)\right|^{2} d t=1 .
\end{aligned}
$$

This property states that the energy of the cross ambiguity function is a constant, independent of the waveforms $u_{m}(t)$ and $u_{m^{\prime}}(t)$. In the special case of $m=m^{\prime}$, this property reduces to the well-known result that the SIMO radar ambiguity function defined in (1) has constant energy [25]. The following property characterizes the energy of the MIMO radar ambiguity function.

4) Property 4: If $\gamma$ is an integer, then

$$
\int_{0}^{1} \int_{0}^{1} \int_{-\infty}^{\infty} \int_{-\infty}^{\infty}\left|\chi\left(\tau, \nu, f, f^{\prime}\right)\right|^{2} d \tau d \nu d f d f^{\prime}=M^{2}
$$

Proof: By using the definition of MIMO radar ambiguity function in (2) and performing appropriate change of variables, we have (10), shown at the bottom of the page. Using
Parserval's theorem and applying Property 3, the above integral reduces to

$\int_{-\infty}^{\infty} \int_{-\infty}^{\infty} \sum_{m=0}^{M-1} \sum_{m^{\prime}=0}^{M-1}\left|\chi_{m, m^{\prime}}(\tau, \nu)\right|^{2} d \tau d \nu=\sum_{m^{\prime}=0}^{M-1} \sum_{m^{\prime}=0}^{M-1} 1=M^{2}$

This property states that when $\gamma$ is an integer, the energy of the MIMO radar ambiguity function is a constant which is independent of the waveforms $\left\{u_{m}(t)\right\}$. For example, whether we choose $\gamma=1$ or $\gamma=N$, the energy of the MIMO radar ambiguity function is the same. Recall that Property 2 states that the integration of MIMO radar ambiguity function along the line $\{(0,0, f, f\}$ is also a constant. This implies that in order to make the ambiguity function sharp around $\{0,0, f, f\}$, we have to spread the energy of the ambiguity function evenly on the available time and bandwidth.

For the case that $\gamma$ is not an integer, we can not directly apply Parserval's theorem. In this case, the energy of the ambiguity function actually depends on the waveforms $\left\{u_{m}(t)\right\}$. However, the following property characterizes the range of the energy of the MIMO radar ambiguity function.

5) Property 5:

$$
\begin{aligned}
\frac{\lfloor\gamma\rfloor^{2}}{\gamma^{2}} M^{2} & \leq \int_{0}^{1} \int_{0}^{1} \int_{-\infty}^{\infty} \int_{-\infty}^{\infty}\left|\chi\left(\tau, \nu, f, f^{\prime}\right)\right|^{2} d \tau d \nu d f d f^{\prime} \\
& \leq \frac{\lceil\gamma\rceil^{2}}{\gamma^{2}} M^{2}
\end{aligned}
$$

where $\lfloor\gamma\rfloor$ is the largest integer $\leq \gamma$, and $\lceil\gamma\rceil$ is the smallest integer $\geq \gamma$.

Proof: Using (10), we have See equation (12), shown at the bottom of the page. Using Parserval's theorem and applying Property 3 , the above value equals

$$
\frac{\lceil\gamma\rceil^{2}}{\gamma^{2}} \int_{-\infty}^{\infty} \int_{-\infty}^{\infty} \sum_{m=0}^{M-1} \sum_{m^{\prime}=0}^{M-1}\left|\chi_{m, m^{\prime}}(\tau, \nu)\right|^{2} d \tau d \nu=\frac{\lceil\gamma\rceil^{2}}{\gamma^{2}} M^{2}
$$

The lower bound can be obtained similarly.

For the case that $\gamma$ is not integer, the energy of the MIMO radar ambiguity function can actually be affected by the waveforms $\left\{u_{m}(t)\right\}$. However, the above property implies that when $\gamma$ is large, the amount by which the energy can be affected by the waveforms is small. Note that the bound provided by this

$$
\int_{0}^{1} \int_{0}^{1} \int_{-\infty}^{\infty} \int_{-\infty}^{\infty}\left|\chi\left(\tau, \nu, f, f^{\prime}\right)\right|^{2} d \tau d \nu d f d f^{\prime}=\frac{1}{\gamma^{2}} \int_{-\infty}^{\infty} \int_{-\infty}^{\infty} \int_{0}^{\gamma} \int_{0}^{\gamma \mid}\left|\sum_{m=0}^{M-1} \sum_{m^{\prime}=0}^{M-1} \chi_{m, m^{\prime}}(\tau, \nu) e^{j 2 \pi\left(f m-f^{\prime} m^{\prime}\right)}\right|^{2} d f d f^{\prime} d \tau d \nu
$$

$$
\int_{0}^{1} \int_{0}^{1} \int_{-\infty}^{\infty} \int_{-\infty}^{\infty}\left|\chi\left(\tau, \nu, f, f^{\prime}\right)\right|^{2} d \tau d \nu d f d f^{\prime} \leq \frac{1}{\gamma^{2}} \int_{-\infty}^{\infty} \int_{-\infty}^{\infty} \int_{0}^{\lceil\gamma\rceil} \int_{0}^{\lceil\gamma\rceil}\left|\sum_{m=0}^{M-1} \sum_{m^{\prime}=0}^{M-1} \chi_{m, m^{\prime}}(\tau, \nu) e^{j 2 \pi\left(f m-f^{\prime} m^{\prime}\right)}\right|^{2} d f d f^{\prime} d \tau d \nu
$$


property is loose when $\gamma$ is small. This is because in (12), we have quantized $\gamma$ in the integration interval in order to apply the Parserval's theorem. However, in order to form a large virtual array and keep the interference rejection ability on the receiver side, the spacings between the transmitting antennas are usually larger than those of the receiving antennas. So $\gamma$ is usually large. Using similar lines of argument as in (12), we can show that when $\gamma$ is not an integer, Property 2 can be replaced with the following property.

6) Property 6:

$$
M \frac{\lfloor\gamma\rfloor}{\gamma} \leq \int_{0}^{1} \chi(0,0, f, f) d f \leq M \frac{\lceil\gamma\rceil}{\gamma} .
$$

The following property characterizes the symmetry of the cross ambiguity function.

7) Property 7:

$$
\chi_{m, m^{\prime}}(-\tau,-\nu)=\chi_{m^{\prime}, m}^{*}(\tau, \nu) e^{-j 2 \pi \nu \tau}
$$

Proof: By the definition of the cross ambiguity function (3) and changing variables, we have

$$
\begin{aligned}
\chi_{m, m^{\prime}}(-\tau,-\nu) & =\int_{-\infty}^{\infty} u_{m}(t) u_{m^{\prime}}^{*}(t-\tau) e^{-j 2 \pi \nu t} d t \\
& =\int_{-\infty}^{\infty} u_{m}(t+\tau) u_{m^{\prime}}^{*}(t) e^{-j 2 \pi \nu(t+\tau)} d t \\
& =\chi_{m^{\prime}, m}^{*}(\tau, \nu) e^{-j 2 \pi \nu \tau} .
\end{aligned}
$$

Using the above property, we can obtain the following property of the MIMO radar ambiguity function.

8) Property 8:

$$
\chi\left(-\tau,-\nu, f, f^{\prime}\right)=\chi^{*}\left(\tau, \nu, f^{\prime}, f\right) e^{-j 2 \pi \nu \tau} .
$$

Proof: Using the definition of the MIMO radar ambiguity function (2) and Property 7, we have

$$
\begin{aligned}
& \chi\left(-\tau,-\nu, f, f^{\prime}\right) \\
& =\sum_{m=0}^{M-1} \sum_{m^{\prime}=0}^{M-1} \chi_{m, m^{\prime}}(-\tau,-\nu) e^{j 2 \pi \gamma\left(f m-f^{\prime} m^{\prime}\right)} \\
& =\sum_{m=0}^{M-1} \sum_{m^{\prime}=0}^{M-1} \chi_{m^{\prime}, m}^{*}(\tau, \nu) e^{-j 2 \pi \nu \tau} e^{j 2 \pi \gamma\left(f m-f^{\prime} m^{\prime}\right)} \\
& =\left(\sum_{m=0}^{M-1} \sum_{m^{\prime}=0}^{M-1} \chi_{m^{\prime}, m}(\tau, \nu) e^{j 2 \pi \gamma\left(f^{\prime} m^{\prime}-f m\right)}\right)^{*} e^{-j 2 \pi \nu \tau} \\
& =\chi^{*}\left(\tau, \nu, f^{\prime}, f\right) e^{-j 2 \pi \nu \tau} .
\end{aligned}
$$

This property implies that when we design the waveform, we only need to focus on the region $\left\{\left(\tau, \nu, f, f^{\prime}\right) \mid \tau \geq 0\right\}$ or the region $\left\{\left(\tau, \nu, f, f^{\prime}\right) \mid f \geq f^{\prime}\right\}$ of the MIMO radar ambiguity function. For example, given two spatial frequencies $f$ and $f^{\prime}$ it is sufficient to study only $\chi\left(\tau, \nu, f, f^{\prime}\right)$ because the function $\chi\left(\tau, \nu, f^{\prime}, f\right)$ can be deduced from the symmetry property. The following property characterizes the cross ambiguity function of the linear frequency modulation (LFM) signal.

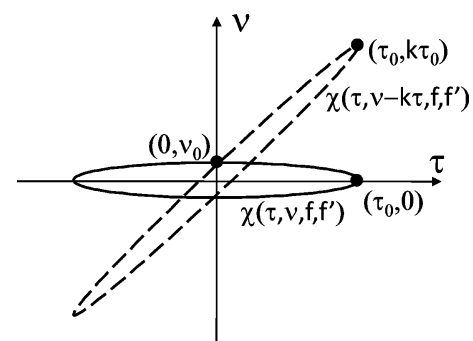

Fig. 3. Illustration of the LFM shearing.

9) Property 9: Define

$$
u_{m}^{L F M}(t) \triangleq u_{m}(t) e^{j \pi k t^{2}} .
$$

$$
\begin{aligned}
& \text { If } \chi_{m, m^{\prime}}(\tau, \nu)=\int_{-\infty}^{\infty} u_{m}(t) u_{m^{\prime}}^{*}(t+\tau) e^{j 2 \pi \nu t} d t \text { then } \\
& \chi_{m, m^{\prime}}^{L F M}(\tau, \nu) \triangleq \int_{-\infty}^{\infty} u_{m}^{L F M}(t)\left(u_{m^{\prime}}^{L F M}(t+\tau)\right)^{*} e^{j 2 \pi \nu t} d t \\
& =\chi_{m, m^{\prime}}(\tau, \nu-k \tau) e^{-j \pi k \tau^{2}} \text {. }
\end{aligned}
$$

Proof: From direct calculation, we have

$$
\begin{aligned}
\chi_{m, m^{\prime}}^{L F M}(\tau, \nu) & =\int_{-\infty}^{\infty} u_{m}(t) u_{m^{\prime}}^{*}(t+\tau) \cdot e^{j \pi k\left(-2 t \tau-\tau^{2}\right)} e^{j 2 \pi \nu t} d t \\
& =\chi_{m, m^{\prime}}(\tau, \nu-k \tau) e^{-j \pi k \tau^{2}} .
\end{aligned}
$$

This property says that linear frequency modulation shears off the cross ambiguity function. We use this property to obtain the following result for the MIMO radar ambiguity function.

10) Property 10: If $\chi\left(\tau, \nu, f, f^{\prime}\right)=\sum_{m=0}^{M-1} \sum_{m^{\prime}=0}^{M-1}$ $\times \chi_{m, m^{\prime}}(\tau, \nu) e^{j 2 \pi \gamma\left(f m-f^{\prime} m^{\prime}\right)}$, then

$$
\begin{aligned}
\chi^{L F M}\left(\tau, \nu, f, f^{\prime}\right) & \triangleq \sum_{m=0}^{M-1} \sum_{m^{\prime}=0}^{M-1} \chi_{m, m^{\prime}}^{L F M}(\tau, \nu) e^{j 2 \pi \gamma\left(f m-f^{\prime} m^{\prime}\right)} \\
& =\chi\left(\tau, \nu-k \tau, f, f^{\prime}\right) e^{-j \pi k \tau^{2}}
\end{aligned}
$$

We omit the proof because this property can be easily obtained by just applying Property 9 . This property states that adding LFM modulations shears off the MIMO radar ambiguity function. This shearing can improve the range resolution because it compresses the ambiguity function along the direction $(\tau, 0, f, f)$ [25]. Fig. 3 illustrates contours of constants $\chi\left(\tau, \nu, f, f^{\prime}\right)$ and $\chi\left(\tau, \nu-k \tau, f, f^{\prime}\right)$ with some fixed $f$ and $f^{\prime}$. One can observe that the delay resolution has been improved after the LFM shearing.

To summarize, Properties 1 to 6 characterize the signal component and the energy of the ambiguity function. They imply that if we attempt to squeeze the ambiguity function to the line $\{0,0, f, f\}$, the signal component cannot go arbitrarily high. Also, if we attempt to eliminate some unwanted peaks in the ambiguity function, the energy will reappear somewhere else. Property 8 suggests that it is sufficient to study only half of the ambiguity function $(\tau \geq 0)$. Properties 9 and 10 imply that the LFM modulation shears the ambiguity function. Therefore, it improves the resolution along the range dimension. 


\section{Pulse MimO RADAR Ambiguity Function}

In this paper, we consider the waveform design problem for the pulse waveforms generated by frequency-hopping codes. In this section, we derive the MIMO radar ambiguity function for the case when the waveform $u_{m}(t)$ consists of the shifted versions of a shorter waveform $\phi_{m}(t)$. In this case, the pulse design problem becomes choosing the waveform $\phi_{m}(t)$ to obtain a good MIMO ambiguity function $\chi\left(\tau, \nu, f, f^{\prime}\right)$. Therefore, it is important to study the relation between the MIMO ambiguity function and the pulse $\phi_{m}(t)$. Since modulation and scalar multiplication will not change the shape of the ambiguity function, for convenience, we write the transmitted signals as

$$
u_{m}(t)=\sum_{l=0}^{L-1} \phi_{m}\left(t-T_{l}\right)
$$

Fig. 4 illustrates the transmitted pulse waveform. Note that the duration of $\phi_{m}(t)$, namely $T_{\phi}$, is small enough such that $T_{\phi} \ll \min _{l, l^{\prime}}\left(\left|T_{l}-T_{l^{\prime}}\right|\right)$. To obtain the relation between $\phi_{m}(t)$ and the MIMO ambiguity function $\chi\left(\tau, \nu, f, f^{\prime}\right)$, we first derive the cross ambiguity function. Using (3) and (18) and changing variables, the cross ambiguity function can be expressed as

$$
\begin{aligned}
& \chi_{m, m^{\prime}}(\tau, \nu) \\
& =\sum_{l^{\prime}=0}^{L-1} \sum_{l=0}^{L-1} \int_{-\infty}^{\infty} \phi_{m}(t) \phi_{m^{\prime}}^{*}\left(t+T_{l}-T_{l^{\prime}}+\tau\right) e^{j 2 \pi \nu\left(t+T_{l}\right)} d t \\
& =\sum_{l^{\prime}=0}^{L-1} \sum_{l=0}^{L-1} \chi_{m, m^{\prime}}^{\phi}\left(\tau+T_{l}-T_{l^{\prime}}, \nu\right) e^{j 2 \pi \nu T_{l}}
\end{aligned}
$$

where $\chi_{m, m^{\prime}}^{\phi}(\tau, \nu)$ is defined as the cross ambiguity function of the pulses $\phi_{m}(t)$ and $\phi_{m^{\prime}}(t)$, that is

$$
\chi_{m, m^{\prime}}^{\phi}(\tau, \nu)=\int_{0}^{T_{\phi}} \phi_{m}(t) \phi_{m^{\prime}}^{*}(t+\tau) e^{j 2 \pi \nu t} d t .
$$

We assume that the Doppler frequency $\nu$ and the support of pulse $T_{\phi}$ are both small enough such that $T_{\phi} \nu \approx 0$. This means the Doppler frequency envelope remains approximately constant within the pulse. Such an assumption is usually made in pulse Doppler processing [27]. So the above the equation becomes

$$
\chi_{m, m^{\prime}}^{\phi}(\tau, \nu) \approx \int_{0}^{T_{\phi}} \phi_{m}(t) \phi_{m^{\prime}}^{*}(t+\tau) d t \triangleq r_{m, m^{\prime}}^{\phi}(\tau)
$$

where $r_{m, m^{\prime}}^{\phi}(\tau)$ is the cross correlation between $\phi_{m}(t)$ and $\phi_{m^{\prime}}(t)$. Thus, the cross ambiguity function reduces to the cross correlation function and it is no longer a function of Doppler frequency $\nu$. Substituting the above result into (19), we obtain

$$
\chi_{m, m^{\prime}}(\tau, \nu) \approx \sum_{l^{\prime}=0}^{L-1} \sum_{l=0}^{L-1} r_{m, m^{\prime}}^{\phi}\left(\tau+T_{l}-T_{l^{\prime}}\right) e^{j 2 \pi \nu T_{l}} .
$$

For values of the delay $\tau$ satisfying $|\tau|<\min _{l, l^{\prime}}\left(\left|T_{l}-T_{l^{\prime}}\right|\right)-$ $T_{\phi}$, the shifted correlation function satisfies

$$
r_{m, m^{\prime}}^{\phi}\left(\tau+T_{l}-T_{l^{\prime}}\right)=\int_{0}^{T_{\phi}} \phi_{m}(\tau) \phi_{m^{\prime}}^{*}\left(t+\tau+T_{l}-T_{l^{\prime}}\right) d t=0
$$

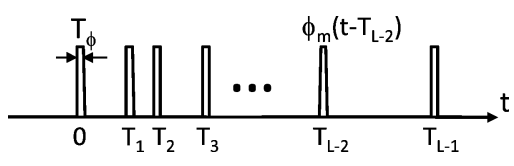

Fig. 4. Illustration of the pulse waveform.

when $l \neq l^{\prime}$. For $|\tau| \geq \min _{l, l^{\prime}}\left(\left|T_{l}-T_{l^{\prime}}\right|\right)-T_{\phi}$, the response in the ambiguity function is created by the second trip echoes. This ambiguity is called range folding. In this paper, we assume the pulse repetition frequency (PRF) is low enough so that no reflections occur at these second trip ranges. We will focus on the ambiguity function only when $|\tau|<\min _{l, l^{\prime}}\left(\left|T_{l}-T_{l^{\prime}}\right|\right)-T_{\phi}$. In this case, we have

$$
\chi_{m, m^{\prime}}(\tau, \nu) \approx r_{m, m^{\prime}}^{\phi}(\tau) \sum_{l=0}^{L-1} e^{j 2 \pi \nu T_{l}}
$$

Notice that the Doppler processing is separable from the correlation function. This is because of the assumption that the duration of the pulses $T_{\phi}$ and the Doppler frequency $\nu$ are small enough so that $\nu T_{\phi} \approx 0$. This implies that the choice of the waveforms $\left\{\phi_{m}(t)\right\}$ does not affect the Doppler resolution. Using the definition of MIMO ambiguity function (2), we have

$\chi\left(\tau, \nu, f, f^{\prime}\right)=\sum_{m=0}^{M-1} \sum_{m^{\prime}=0}^{M-1} r_{m, m^{\prime}}^{\phi}(\tau) e^{j 2 \pi\left(f m-f^{\prime} m^{\prime}\right) \gamma} \cdot \sum_{l=0}^{L-1} e^{j 2 \pi \nu T_{l}}$

for $|\tau|<\min _{l, l^{\prime}}\left(\left|T_{l}-T_{l^{\prime}}\right|\right)-T_{\phi}$.

The preceding analysis clearly shows how the problem of waveform design should be approached. The MIMO ambiguity function depends on the cross correlation functions $r_{m, m^{\prime}}^{\phi}(\tau)$. Also, the pulses $\left\{\phi_{m}(t)\right\}$ only affect the range and spatial resolution. They do not affect the Doppler resolution. Therefore, to obtain a sharp ambiguity function, we should design the pulses $\left\{\phi_{m}(t)\right\}$ such that the function

$$
\Omega\left(\tau, f, f^{\prime}\right) \triangleq \sum_{m=0}^{M-1} \sum_{m^{\prime}=0}^{M-1} r_{m, m^{\prime}}^{\phi}(\tau) e^{j 2 \pi\left(f m-f^{\prime} m^{\prime}\right) \gamma}
$$

is sharp around the line $\left\{\left(\tau, f, f^{\prime}\right) \mid \tau=0, f=f^{\prime}\right\}$. For $M=$ 1 , the signal design problem reduces to the special case of the SIMO radar. In this case, (22) reduces to the autocorrelation function

$$
\Omega\left(\tau, f, f^{\prime}\right)=r_{0,0}^{\phi}(\tau)
$$

Thus in the SIMO radar case, the signal design problem is to generate a pulse with a sharp autocorrelation. The linear frequency modulation (LFM) signal is an example which has a sharp autocorrelation [25]. Besides its sharp autocorrelation function, the LFM pulse can be conveniently generated and it has constant modulus. These reasons make the LFM signal a very good candidate in a pulse repetition radar system. For the MIMO radar case which satisfies $M>1$, we need to consider not only the autocorrelation functions but also the cross correlation functions between pulses such that $\Omega\left(\tau, f, f^{\prime}\right)$ can be sharp. 


\section{FREQUENCY-HoPPING PULSES}

Instead of directly designing the pulses, we can impose some structures on the pulses and design the parameters of the pulses. As an example of this idea, we now consider the pulse generated by frequency-hopping codes. In this section, we derive the MIMO radar ambiguity function of the frequency-hopping pulses. These pulses have the advantage of constant modulus. The frequency-hopping pulses can be expressed as

$$
\phi_{m}(t)=\sum_{q=0}^{Q-1} e^{j 2 \pi c_{m, q} \Delta f t} s(t-q \Delta t)
$$

where

$$
s(t) \triangleq \begin{cases}1, & t \in[0, \Delta t) \\ 0, & \text { otherwise }\end{cases}
$$

$c_{m, q} \in\{0,1, \ldots, K-1\}$ is the frequency-hopping code, and $Q$ is the length of the code. The duration of the pulse is $T_{\phi}=Q \Delta t$, and the bandwidth of the pulses is approximately

$$
B W_{\phi} \approx(K-1) \Delta f+\frac{1}{\Delta t} .
$$

In this paper, we are interested in the design of orthogonal waveforms. To maintain orthogonality, the code $\left\{c_{m, q}\right\}$ could be constrained to satisfy

$$
c_{m, q} \neq c_{m^{\prime}, q}, \text { for } m \neq m^{\prime}, \forall q \quad \Delta t \Delta f=1 .
$$

Now instead of directly designing the pulses $\phi_{m}(t)$, the signal design problem becomes designing the code $c_{m, q}$ for $m=$ $0,1, \ldots, M-1$ and $q=0,1, \ldots, Q-1$. Recall that our goal is to design the transmitted signals such that the function $\Omega\left(\tau, f, f^{\prime}\right)$ in (22) is sharp (as explained in Section V). So, we are interested in the expression for the function $\Omega\left(\tau, f, f^{\prime}\right)$ in terms of $\left\{c_{m, q}\right\}$. To compute the function $\Omega\left(\tau, f, f^{\prime}\right)$, we first compute the cross correlation function $r_{m, m}^{\phi}(\tau)$. By using (23) and (20), this can be expressed as (25), shown at the bottom of the page, where $\chi^{\text {rect }}(\tau, \nu)$ is the SIMO ambiguity function of the rectangular pulse $s(t)$, given by

$$
\begin{aligned}
& \chi^{\mathrm{rect}}(\tau, \nu) \\
& \triangleq \int_{0}^{\Delta t} s(t) s(t+\tau) e^{j 2 \pi \nu} d t \\
& = \begin{cases}\frac{\Delta t-|\tau|}{\Delta t} \operatorname{sinc}(\nu(\Delta t-|\tau|)) e^{j \pi \nu(\tau+\Delta t)}, & \text { if }|\tau|<\Delta t \\
0, & \text { otherwise. }\end{cases}
\end{aligned}
$$

Substituting (25) into (22), we obtain the equation shown at the bottom of the page. Define $\tau=k \Delta t+\eta$, where $|\eta|<\Delta t$. By using the fact that $\chi^{\text {rect }}(\tau, \nu)=0$ when $|\tau|>\Delta t$, the above equation can be further simplified as (27) shown at the bottom of the page. The next step is to choose the frequency-hopping code $\left\{c_{m, q}\right\}$ such that the function $\Omega\left(\tau, f, f^{\prime}\right)$ is sharp around $\{0, f, f\}$. We will discuss this in the following section.

\section{OptimizATION OF THE FREQuenCY-HopPing CODES}

In this section, we introduce an algorithm to search for frequency-hopping codes which generate good MIMO ambiguity functions. By using (22) and the orthogonality of the waveforms, we have

$$
\Omega(0, f, f)=\sum_{m, m^{\prime}=0}^{M-1} \delta_{m, m^{\prime}} e^{j 2 \pi f \gamma\left(m-m^{\prime}\right)}=M .
$$

So, we know that the function $\Omega(\tau, f, f)$ is a constant along the line $\{0, f, f\}$, no matter what codes are chosen. To obtain good system resolutions, we need to eliminate the peaks in $\left|\Omega\left(\tau, f, f^{\prime}\right)\right|$ which are not on the line $\{0, f, f\}$. This can be done by imposing a cost function which puts penalties on these peak values. This forces the energy of the function $\Omega\left(\tau, f, f^{\prime}\right)$ to be evenly spread in the delay and angular dimensions. As an example of this, we minimize the $p$-norm of the function

$$
r_{m, m^{\prime}}^{\phi}(\tau)=\sum_{q=0}^{Q-1} \sum_{q^{\prime}=0}^{Q-1} \chi^{\mathrm{rect}}\left(\tau-\left(q^{\prime}-q\right) \Delta t,\left(c_{m, q}-c_{m^{\prime}, q^{\prime}}\right) \Delta f\right) \cdot e^{j 2 \pi \Delta f\left(c_{m, q}-c_{m^{\prime}, q^{\prime}}\right) q \Delta t} e^{j 2 \pi \Delta f c_{m^{\prime}, p^{\prime}} \tau}
$$

$$
\Omega\left(\tau, f, f^{\prime}\right)=\sum_{m, m^{\prime}=0}^{M-1} \sum_{q, q^{\prime}=0}^{Q-1} \chi^{\mathrm{rect}}\left(\tau-\left(q^{\prime}-q\right) \Delta t,\left(c_{m, q}-c_{m^{\prime}, q^{\prime}}\right) \Delta f\right) \cdot e^{j 2 \pi \Delta f\left(c_{m, q}-c_{m^{\prime}, q^{\prime}}\right) q \Delta t} e^{j 2 \pi \Delta f c_{m^{\prime}, q^{\prime}} \tau} e^{j 2 \pi\left(f m-f^{\prime} m^{\prime}\right)} .
$$

$\Omega\left(k \Delta t+\eta, f, f^{\prime}\right)=\sum_{m, m^{\prime}=0}^{M-1} \sum_{q=0}^{Q-1} \chi^{\mathrm{rect}}\left(\eta,\left(c_{m, q}-c_{m^{\prime}, q+k}\right) \Delta f\right) \cdot e^{j 2 \pi \Delta f c_{m^{\prime}, q+k}(k \Delta t+\eta)} e^{j 2 \pi \Delta f\left(c_{m, q}-c_{m^{\prime}, q+k}\right) q \Delta t} \cdot e^{j 2 \pi\left(f m-f^{\prime} m^{\prime}\right) \gamma}$. 
$\Omega\left(\tau, f, f^{\prime}\right)$. The corresponding optimization problem can be expressed as

$$
\begin{array}{r}
\min _{\mathbf{C}} f_{p}(\mathbf{C}) \\
\text { subject to } \mathbf{C} \in\{0,1, \ldots, K-1\}^{M Q} \\
c_{m, q} \neq c_{m^{\prime}, q}, \text { for } m \neq m^{\prime}
\end{array}
$$

where

$$
f_{p}(\mathbf{C}) \triangleq \int_{-\infty}^{\infty} \int_{0}^{1} \int_{0}^{1}\left|\Omega\left(\tau, f, f^{\prime}\right)\right|^{p} d f d f^{\prime} d \tau
$$

and $c_{m, q}$ denotes the $(m, q)$ entry of the matrix C. Note that a greater $p$ imposes more penalty on the higher peaks. The feasible set of this problem is a discrete set. It is known that the simulated annealing algorithm is very suitable for solving this kind of problems [23]. The simulated annealing algorithm runs a Markov chain Monte Carlo (MCMC) sampling on the discrete feasible set [24]. The transition probability of the Markov chain can be chosen so that the equilibrium of the Markov chain is

$$
\begin{aligned}
\pi_{T}(\mathbf{C}) & =\frac{1}{Z_{T}} \exp \left(\frac{-f_{p}(\mathbf{C})}{T}\right) \\
\text { where } Z_{T} & =\sum_{\mathbf{C}} \exp \left(\frac{-f_{p}(\mathbf{C})}{T}\right) .
\end{aligned}
$$

Here $T$ is a parameter called temperature. By running the MCMC and gradually decreasing the temperature $T$, the generated sample $\mathbf{C}$ will have a high probability to have a small cost function output [23]. In our case, the transition probability from state $\mathbf{C}$ to $\mathbf{C}^{\prime}$ is chosen as

$$
\begin{aligned}
& p\left(\mathbf{C}, \mathbf{C}^{\prime}\right) \\
& = \begin{cases}\frac{1}{d} \min \left(1, \exp \left(\frac{f_{p}(\mathbf{C})-f_{p}\left(\mathbf{C}^{\prime}\right)}{T}\right)\right), & \text { if } \mathbf{C}^{\prime} \sim \mathbf{C} \\
1-\frac{1}{d} \sum_{\mathbf{C}^{\prime \prime} \sim \mathbf{C}} \min \left(1, \exp \left(\frac{f_{p}(\mathbf{C})-f_{p}\left(\mathbf{C}^{\prime \prime}\right)}{T}\right)\right), & \text { if } \mathbf{C}^{\prime}=\mathbf{C} \\
0, & \text { otherwise }\end{cases}
\end{aligned}
$$

where $\mathbf{C}^{\prime} \sim \mathbf{C}$ denotes that $\mathbf{C}^{\prime}$ and $\mathbf{C}$ differ in exactly one element, and $d$ denotes $\left|\left\{\mathbf{C}^{\prime} \mid \mathbf{C}^{\prime} \sim \mathbf{C}\right\}\right|$. It can be shown that the chosen transition probabilities result in the desire equilibrium in (30) [24]. The corresponding MCMC sampling can be implemented as the following algorithm.

Algorithm 1: Given number of waveforms $M$, length of the code $Q$, number of frequencies $K$, initial temperature $T$, and a temperature decreasing ratio $\alpha \in(0,1)$, the code $\mathbf{C} \in\{0,1, \ldots, K-1\}^{M Q}$ can be computed by the following steps:

1. Randomly draw $\mathbf{C}$ from $\{0,1, \ldots, K-1\}^{M Q}$

such that $c_{m q} \neq c_{m^{\prime} q}$ for $m \neq m$.

2. Randomly draw $m$ from $\{0,1, \ldots, M-1\}$

and $q$ from $\{0,1, \ldots, Q-1\}$.
3. Randomly draw $k$ from $\{0,1, \ldots, K-1\} \backslash c_{m q}$.

4. $\mathbf{C}^{\prime} \leftarrow \mathbf{C}, c_{m q}^{\prime} \leftarrow K$.

5. Randomly draw $U$ from $[0,1]$.

6. If $U<\exp \left(\left(f_{p}(\mathbf{C})-f_{p}\left(\mathbf{C}^{\prime}\right)\right) / T\right), \mathbf{C} \leftarrow \mathbf{C}^{\prime}$.

7. If the cost $f_{p}(\mathbf{C})$ is small enough, stop.

else $T \leftarrow \alpha T$ and go to Step 2 .

\section{DESIGN EXAMPLES}

In this section, we present a design example using the proposed method. In this example, we consider a uniform linear transmitting array. The number of transmitted waveforms $M$ equals 4 . The length of the frequency-hopping code $Q$ equals 10 . The number of frequencies $K$ equals 15 . Without loss of generality, we normalize the pulse duration $T_{\phi}$ to be unity. By using (24), we obtain that the time-bandwidth product

$$
\left((K-1) \Delta f+\frac{1}{\Delta t}\right) Q \Delta t=150 .
$$

Note that this implies the maximum number of orthogonal waveform obtainable is $B T=150$ [22]. So, our choice of $M=4$ orthogonal waveforms is well under the theoretical limit. The cost function in (29) can be approximated by a Riemann sum. By applying the symmetry given by Property 8 , we can integrate only the part that has $\tau \geq 0$. Fig. 5 shows the real parts and the spectrograms of the waveforms generated by the proposed algorithm. For comparison Fig. 6 shows the real parts and the spectrograms of orthogonal LFM waveforms. In this example, these LFM waveforms have the form

$$
\phi_{m}(t)=\exp \left(j 2 \pi f_{m, 0} t+j \pi k t^{2}\right)
$$

where $k=100, f_{0,0}=0, f_{1,0}=\lfloor 50 / 3\rfloor, f_{2,0}=\lfloor 100 / 3\rfloor$, and $f_{3,0}=50$. By choosing different initial frequencies, these LFM waveforms can be made orthogonal. These parameters are chosen so that these LFM waveforms occupy the same time duration and bandwidth as the waveforms generated by the proposed method. Fig. 7 shows a result of comparing the functions $\left|\Omega\left(\tau, f, f^{\prime}\right)\right|$. We take samples from the function $\left|\Omega\left(\tau, f, f^{\prime}\right)\right|$ and plot their empirical cumulative distribution function (ECDF). In other words, this figure shows the percentage of samples of $\left|\Omega\left(\tau, f, f^{\prime}\right)\right|$ less than various magnitude. We have normalized the highest peak to $0 \mathrm{~dB}$. The results of the proposed method, randomly generated frequency-hopping codes, and the LFM waveforms are compared in the figure. One can see that the proposed frequency-hopping signals yield fewest undesired peaks among all the waveforms. The video which shows the entire function $\left|\Omega\left(\tau, f, f^{\prime}\right)\right|$ (a plot in $\left(f, f^{\prime}\right)$ plane as a function of time $\left.\tau\right)$ can be viewed from [28]. Fig. 8 shows the cross correlation functions $r_{m, m^{\prime}}^{\phi}(\tau)$ of the waveforms generated by the proposed algorithm. Fig. 9 shows the cross correlation functions $r_{m, m^{\prime}}^{\phi}(\tau)$ of the LFM waveforms. One can observe that for the proposed waveforms, 
(a)
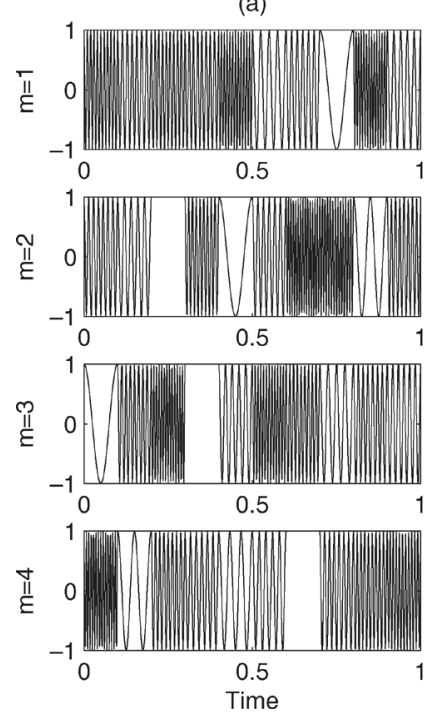

(b)

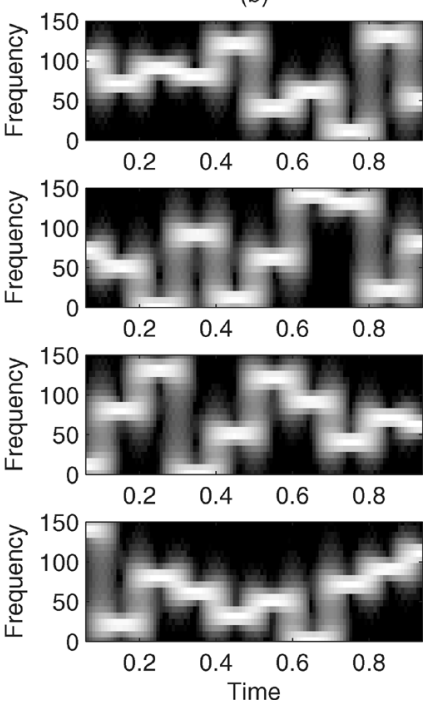

Fig. 5. (a) Real parts and (b) spectrograms of the waveforms obtained by the proposed method. (a)
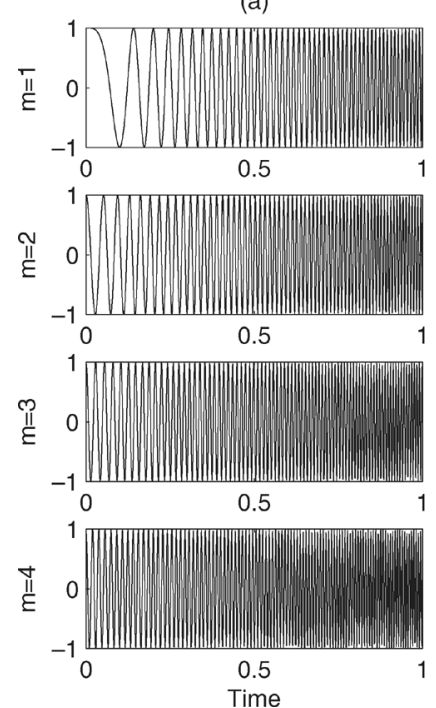

(b)
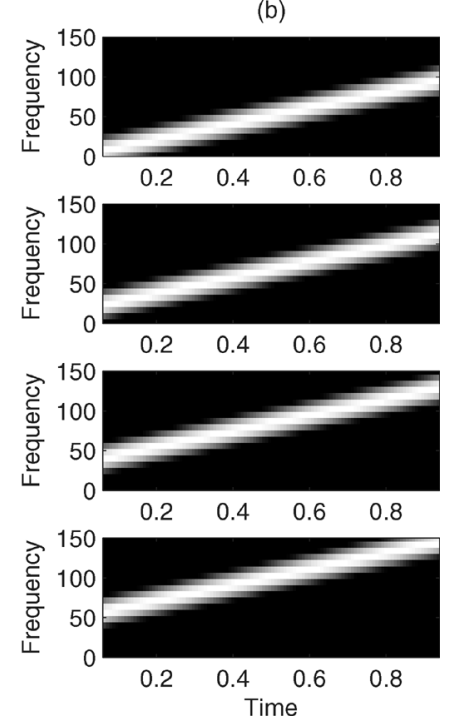

Fig. 6. (a) Real parts and (b) spectrograms of the orthogonal LFM waveforms.

the correlation functions $r_{m, m^{\prime}}^{\phi}(\tau)$ equal to unity when $m=m^{\prime}$ and $\tau=0$. Except at these points, the correlation functions are small everywhere. However, for the LFM waveforms, the correlation functions have several extraneous peaks which also form peaks in the ambiguity function.

\section{CONCLUSION}

In this paper, we have derived several properties of the MIMO radar ambiguity function and the cross ambiguity function. These results are derived for the ULA case. To summarize, Property 1,2, and 6 characterize the MIMO radar ambiguity function along the line $\{(0,0, f, f)\}$. Properties 3,4 , and 5 characterize the energy of the cross ambiguity function and the MIMO radar ambiguity function. These properties imply that we can only spread the energy of the MIMO radar ambiguity function evenly on the available time

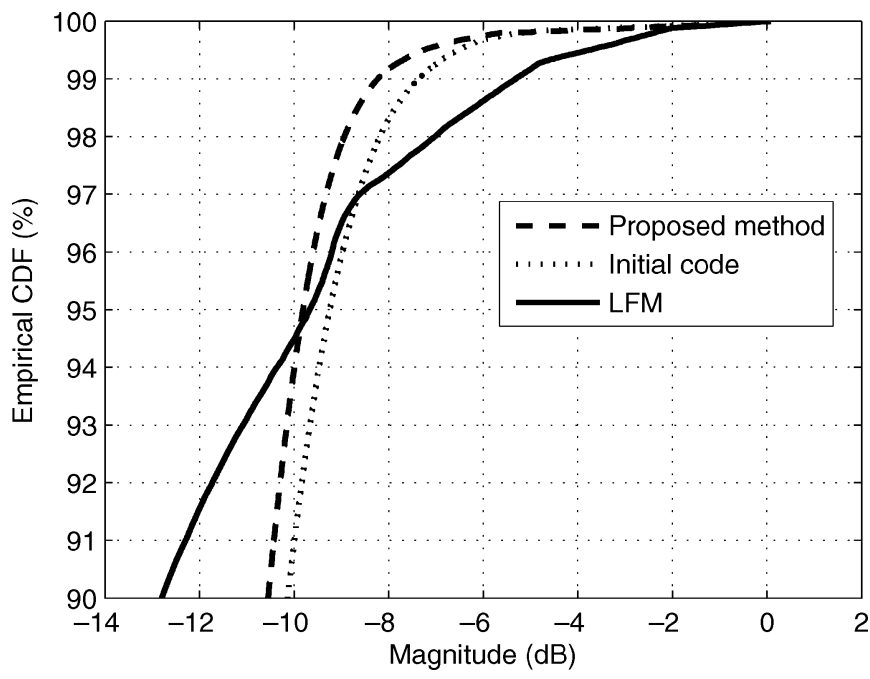

Fig. 7. Empirical cumulative distribution function of $\left|\Omega\left(\tau, f, f^{\prime}\right)\right|$.
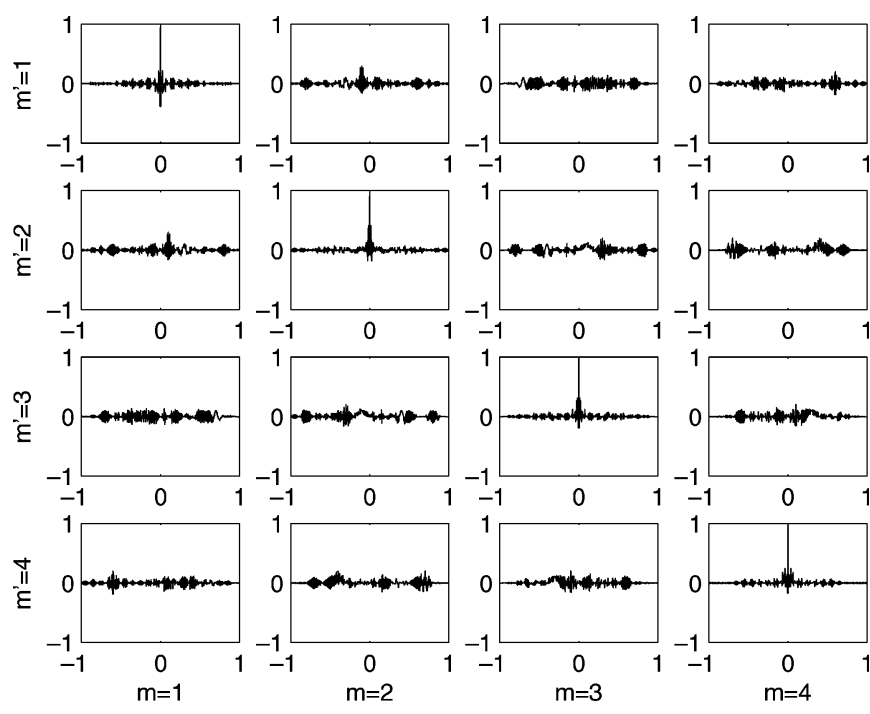

Fig. 8. Cross correlation functions $r_{m, m^{\prime}}^{\phi}(\tau)$ of the waveforms generated by the proposed method.

and bandwidth because the energy is confined. Properties 7 and 8 show the symmetry of the cross ambiguity function and the MIMO radar ambiguity function. These properties imply that when we design the waveform, we only need to focus on the region $\left\{\left(\tau, \nu, f, f^{\prime}\right) \mid \tau \geq 0\right\}$ of the MIMO radar ambiguity function. Properties 9 and 10 show the shear-off effect of the LFM waveform. This shearing improves the range resolution. We have also introduced a waveform design method for MIMO radars. This method is applicable to the case where the transmitted waveforms are orthogonal and consist of multiple shifted narrow pulses. The proposed method applies the simulated annealing algorithm to search for the frequency-hopping codes which minimize the $p$-norm of the ambiguity function. The numerical examples show that the waveforms generated by this method provide better angular and range resolutions than the LFM waveforms which have often been used in the traditional SIMO radar systems. In this paper, we have presented the results only for the case of 

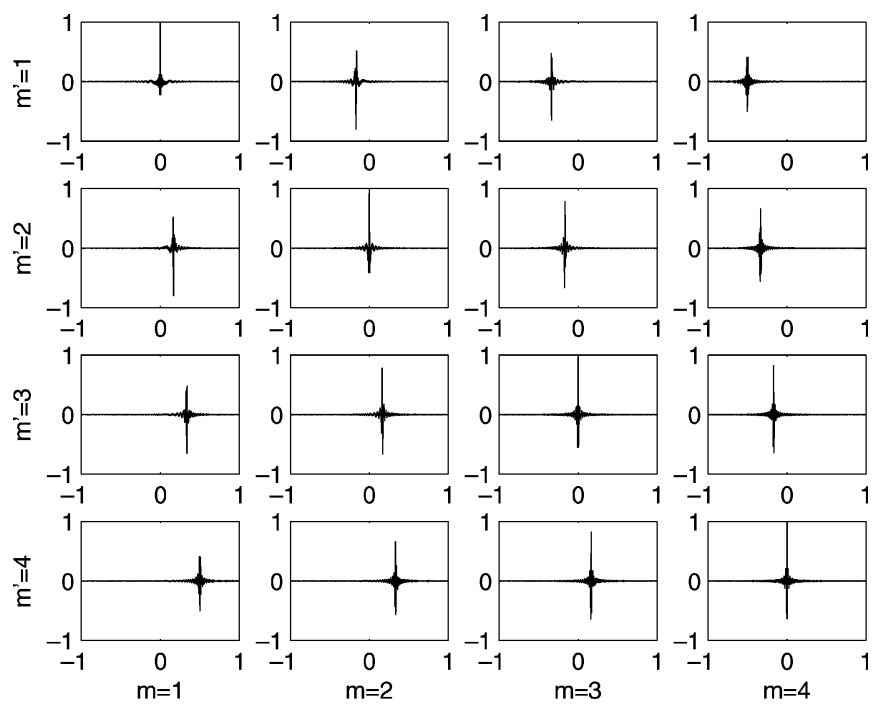

Fig. 9. Cross-correlation functions $r_{m, m^{\prime}}^{\phi}(\tau)$ of the LFM waveforms.

linear arrays. Nevertheless it is possible to further generalize these results for multidimensional arrays.

\section{REFERENCES}

[1] D. J. Rabideau and P. Parker, "Ubiquitous MIMO multifunction digital array radar," in Proc. 37th IEEE Asilomar Conf. Signals, Systems, Computers, Nov. 2003, vol. 1, pp. 1057-1064.

[2] D. W. Bliss and K. W. Forsythe, "Multiple-input multiple-output (MIMO) radar and imaging: Degrees of freedom and resolution," in Proc. 37th IEEE Asilomar Conf. Signals, Systems, Computers, Nov. 2003, vol. 1, pp. 54-59.

[3] E. Fishler, A. Haimovich, R. S. Blum, D. Chizhik, L. J. Cimini, and R. A. Valenzuela, "MIMO radar: An idea whose time has come," in Proc. IEEE Radar Conf., Apr. 2004, pp. 71-78.

[4] E. Fishler, A. Haimovich, R. S. Blum, L. J. Cimini, D. Chizhik, and R. A. Valenzuela, "Performance of MIMO radar systems: Advantages of angular diversity," in Proc. 38th IEEE Asilomar Conf. Signals, Systems, Computers, Nov. 2004, vol. 1, pp. 305-309.

[5] E. Fishler, A. Haimovich, R. S. Blum, L. J. Cimini, D. Chizhik, and R. A. Valenzuela, "Spatial diversity in radars-models and detection performance," IEEE Trans. Signal Process., vol. 54, no. 3, pp. 823-837, Mar. 2006.

[6] G. San Antonio, D. R. Fuhrmann, and F. C. Robey, "MIMO radar ambiguity functions," IEEE J. Sel. Topics Signal Process., vol. 1, pp. 167-177, Jun. 2007

[7] J. Li and P. Stoica, "MIMO radar with colocated antennas," IEEE Signal Process. Mag., vol. 24, no. 5, pp. 106-114, Sep. 2007.

[8] J. Li, P. Stoica, L. Xu, and W. Roberts, "On parameter identifiability of MIMO radar,” IEEE Signal Process. Lett., vol. 14, no. 12, pp. 968-971, Dec. 2007.

[9] C. Y. Chen and P. P. Vaidyanathan, "MIMO radar space-time adaptive processing using prolate spheroidal wave functions," IEEE Trans. on Signal Processing, to be published.

[10] V. F. Mecca, D. Ramakrishnan, and J. L. Krolik, "MIMO radar space-time adaptive processing for multipath clutter mitigation," in Proc. IEEE Workshop Sensor Array Multichannel Signal Processing, Jul. 2006, pp. 249-253.

[11] D. R. Fuhrmann and G. S. Antonio, "Transmit beamforming for MIMO radar systems using partial signal correlation," in Proc. 38th IEEE Asilomar Conf. Signals, Systems, Computers, Nov. 2004, pp. 295-299.

[12] P. Stoica, J. Li, and Y. Xie, "On probing signal design for MIMO radar," IEEE Trans. Signal Process., vol. 55, no. 8, pp. 4151-4161, Aug. 2007.

[13] B. Friedlander, "Waveform design for MIMO radars," IEEE Trans. Aerosp. Electron. Syst., vol. 43, pp. 1227-1238, Jul. 2007.

[14] Y. Yang and R. S. Blum, "MIMO radar waveform design based on mutual information and minimum mean-square error estimation," IEEE Trans. Aerosp. Electron. Syst., vol. 43, no. 1, pp. 330-343, Jan. 2007.
[15] Y. Yang and R. S. Blum, "Minimax robust MIMO radar waveform design,” IEEE J. Sel. Topics Signal Process., vol. 1, no. 1, pp. 147-155, Jun. 2007.

[16] A. Leshem, O. Naparstek, and A. Nehorai, "Information theoretic adaptive radar waveform design for multiple extended targets," IEEE J. Sel. Topics Signal Process., vol. 1, no. 1, pp. 42-55, Jun. 2007.

[17] M. R. Bell, "Information theory and radar waveform design," IEEE Trans. Inf. Theory, vol. 39, no. 5, pp. 1578-1597, Sep. 1993.

[18] G. J. Frazer, B. A. Johnsone, and Y. I. Abramovich, "Orthogonal waveform support in MIMO HF OTH radars," in Proc. Int. Conf. Waveform Diversity Design, Jun. 2007, pp. 423-427.

[19] S. V. Maric and E. L. Titlebaum, "A class of frequency hop codes with nearly ideal characteristics for use in multiple-access spread-spectrum communications and radar and sonar systems," IEEE Trans. Commun., vol. 40, no. 9, pp. 1442-1447, Sep. 1992.

[20] J. P. Costas, "A study of a class of detection waveforms having nearly ideal range-Doppler ambiguity properties," Proc. IEEE, vol. 72, pp. 996-1009, Aug. 1984.

[21] S. W. Golomb and H. Taylor, "Construction and properties of Costas arrays," Proc. IEEE, vol. 72, pp. 1143-1163, Sep. 1984.

[22] M. Petrich, "On the number of orthogonal signals which can be placed in a WT-product," J. Soc. Ind. Appl. Math., vol. 11, no. 4, pp. 936-940, Dec. 1963.

[23] S. Kirkpatrick, C. D. Gelatt, and M. P. Vecchi, "Optimization by simulated annealing," Science, vol. 220, no. 4598, pp. 671-680, 1983

[24] N. Metropolis, A. W. Rosenbluth, M. N. Rosenbluth, A. H. Teller, and E. Teller, "Equations of state calculations by fast computing machines," J. Chem. Phys., vol. 21, no. 6, pp. 1087-1092, 1953.

[25] N. Levanon and E. Mozeson, Radar Signals. New York: Wiley-IEEE Press, 2004

[26] A. V. Oppenheim and R. W. Schafer, Discrete-Time Signal Processing. Englewood Cliffs, NJ: Prentice-Hall, 1998.

[27] M. A. Richards, Fundamentals of Radar Signal Processing. New York: McGraw-Hill, 2005.

[28] Ambiguity function video [Online]. Available: http://www.systems.caltech.edu/cyc/amb-fun.htm

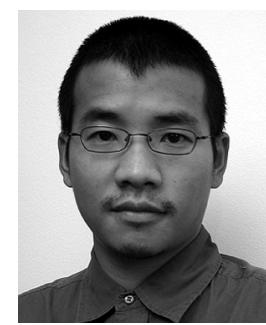

Chun-Yang Chen (S'06) was born in Taipei, Taiwan. He received the B.S. degree in electrical engineering and the M.S. degree in communication engineering both from National Taiwan University, Taiwan, in 2000 and 2002, respectively. He is currently working towards the $\mathrm{Ph}$.D. degree in electrical engineering at the California Institute of Technology, Pasadena.

His interests currently include signal processing in MIMO communications, ultra-wideband communications, and radar applications.

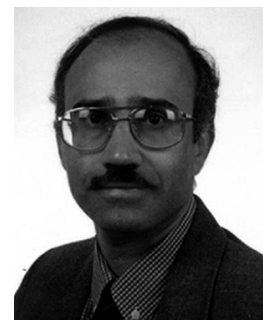

P.P. Vaidyanathan (S'80-M'83-SM'88-F'91) was born in Calcutta, India, on October 16, 1954. He received the B.Sc. (Hons.) degree in physics and the B.Tech. and M.Tech. degrees in radiophysics and electronics, all from the University of Calcutta, India, in 1974, 1977, and 1979, respectively, and the $\mathrm{Ph} . \mathrm{D}$. degree in electrical and computer engineering from the University of California at Santa Barbara in 1982.

He was a Postdoctoral Fellow at the University of California, Santa Barbara, from September 1982 to March 1983. In March 1983, he joined the Electrical Engineering Department of the California Institute of Technology, Pasadena, as an Assistant Professor, where he has been Professor of electrical engineering since 1993. He has authored a number of papers in IEEE journals and is the author of the book Multirate Systems and Filter Banks (Prentice-Hall, 1993). He has written several chapters for various signal processing handbooks. His main research interests are in digital signal processing, multirate systems, wavelet transforms, and signal processing for digital communications.

Dr. Vaidyanathan served as Vice-Chairman of the Technical Program Committee for the 1983 IEEE International Symposium on Circuits and Systems and as the Technical Program Chairman for the 1992 IEEE International symposium on Circuits and Systems. He was an Associate Editor for the IEEE TRANSACTIONS ON CIRCUITS AND SYSTEMS from 1985 to 1987, and is currently an Associate Editor for IEEE SigNAL PROCESSING LETTERS, and a consulting 
editor for the journal Applied and Computational Harmonic Analysis. He was a Guest Editor in 1998 for special issues of the IEEE TRANSACTIONS ON SIGNAL PROCESSING and the IEEE TRANSACTIONS ON CIRCUITS AND SYSTEMS II, on the topics of filter banks, wavelets, and subband coders. He was a recipient of the Award for Excellence in Teaching at the California Institute of Technology for the years 1983-1984, 1992-1993, and 1993-1994. He also received the NSF's Presidential Young Investigator award in 1986. In 1989, he received the IEEE ASSP Senior Award for his paper on multirate perfect-reconstruction filter banks. In 1990, he was recipient of the S. K. Mitra Memorial Award from the Institute of Electronics and Telecommunications Engineers, India, for his joint paper in the IETE journal. He was also the coauthor of a paper on linear-phase perfect reconstruction filter banks in the IEEE Signal Processing
Transactions, for which the first author (T. Nguyen) received the Young Outstanding Author Award in 1993. He received the 1995 F. E. Terman Award of the American Society for Engineering Education, sponsored by Hewlett Packard Company, for his contributions to engineering education, especially the book Multirate systems and filter banks published by Prentice Hall in 1993. He has given several plenary talks, including at the SAMPTA 2001, EUSIPCO 1998, SPCOM 1995, and Asilomar 1988 conferences on signal processing. He has been chosen a Distinguished Lecturer for the IEEE Signal Processing Society for the year 1996-1997. In 1999, he was chosen to receive the IEEE CAS Society's Golden Jubilee Medal. He is a recipient of the IEEE Signal Processing Society's Technical Achievement Award for the year 2002. 